\title{
El Metronidazol en los agrandamientos gingivales secundarios a Ciclosporina $\mathbf{A}$.
}

\author{
MESA FL * \\ OSUNA $\bar{A} * *$ \\ ANEIROS J *** \\ BRAVO J ** \\ JUNCO $\mathbf{P}$ * \\ DEL MORAL RG *** \\ O'VALLE $\mathbf{F} * * *$
}

\begin{abstract}
Mesa FL, Osuna A, Aneiros J, Bravo J, Junco P, Del Moral RG, O'Valle F. Implantes con grabado ácido y pasivado químico: estudio preliminar sobre carga precoz. Av Periodon Implantol. 2002; 14, 2: 69-74.
\end{abstract}

\section{RESUMEN}

Introducción: El agrandamiento gingival (AG) secundario a fármacos continúa siendo un problema importante para los periodoncistas. Han sido comunicadas remisiones parciales o totales del AG tras tratamientos cortos con diversos antibióticos.

Métodos: Hemos realizado un estudio aleatorio controlado a doble ciego para determinar el efecto del tratamiento con metronidazol durante siete días ( $250 \mathrm{mg} / 3$ veces al día) sobre el AG incipiente secundario a ciclosporina $A$ en pacientes adultos trasplantados renales. La valoración cuantitativa del AG se realizó semiautomáticamente con técnicas de Análisis Digital de Imagen.

Resultados: En ninguno de los 26 pacientes incluidos en el estudio se obtuvo la remisión completa del AG al finalizar el estudio. El índice de AG obtenido a tiempo cero fue en los pacientes tratados con metronidazol (grupo MNZ) $0.90 \pm 0.16$ y en los controles (grupo placebo) $1.11 \pm 0.33$. Transcurridos 30 días de evolución el índice de AG en el grupo MNZ no se modificó $(0.92 \pm 0.28)$ y en los pacientes que tomaron placebo se incrementó ligeramente ( $1.18 \pm 0.3$ ), aunque sin llegar a ser estadísticamente significativo. El índice AG disminuyó en el $54.4 \%$ de los pacientes del grupo MNZ, mientras que sólo lo hizo en el $23.1 \%$ en el grupo placebo. A pesar de que no existieron diferencias en el índice de AG entre los dos grupo al principio del ensayo al finalizar el mismo el mayor incremento del IAG en el grupo placebo dió como resultado una diferencia estadísticamente significativa (MNZ $0.92 \pm 0.28$ vs placebo $1.18 \pm 0.3, p<0.05$ t de Student), diferencia que se acentuó cuando se compararón sólo los pacientes de ambos grupos con AG clínico grado 1 ó 2 (MNZ $0.96 \pm 0.31$ vs placebo $1.24 \pm$ $0.31, \mathrm{t}$ de Student $\mathrm{p}<0.05$ ).

Conclusión: Los resultados del presente estudio indican que el tratamiento con metronidazol durante siete días no induce la regresión completa de los agrandamientos gingivales producidos por CsA, pero puede frenar parcialmente el curso clínico del AG, posiblemente al reducir la sobreinfección bacteriana y la inflamación gingival.

\section{PALABRAS CLAVE}

Metronidazol, ciclosporina A, sobrecrecimiento gingival.

\footnotetext{
* Departamento de Periodoncia, Facultad de Odontología, Universidad de Granada.

** Departamento de Nefrologia, Hospital Universitario Virgen de las Nieves de Granada.

*** Departamento de Anatomía Patológica, Hospital Clínico Universitario, Facultad de Medicina. Universidad de Granada.
} 


\section{INTRODUCCIÓN}

El agrandamiento gingival (AG) es una complicación ampliamente documentada como efecto secundario del tratamiento con ciclosporina $A(\mathrm{Cs} A)$, tratada con medidas de higiene para control de placa bacteriana y con gingivectomías parciales en los casos severos (1).

Aunque la patogenia y cambios histopatológicos del AG inducido por CsA están por establecer definitivamente, el AG clínicamente es la combinación de dos componentes histopatológicos principales: Incremento de la fibrosis determinado por el fármaco y lesión inflamatoria debida a la placa bacteriana dental $(2,3)$.

En los últimos años se ha comunicado la utilidad del metronidazol o azitromicina en tratamientos de corta duración en la remisión de los AG inducidos por fármacos (4-9). El MNZ es particularmente activo frente a anaeróbios asociados a periodontitis, en especial espiroquetas (10), y frente A. Actinomycetemcomitans presentes en la placa bacteriana (11). Asociado con espiramicina, MNZ adquiere una alta concentración y vida media en el fluido crevicular gingival, importante para la eliminación de bacterias periodontopatógenas $(3,12)$.

Los resultados publicados sobre el tratamiento antibiótico del AG inducido por CsA no son totalmente consistentes. Wong y colaboradores (4) obtuvieron la remisión completa del AG en cuatro mujeres jóvenes trasplantadas renales después del tratamiento con MNZ a dosis de $1.2 \mathrm{~g} /$ día durante una semana. Más recientemente, Aufircht y colaboradores, informaron sobre la ausencia de mejoría del AG inducido por CsA en pacientes pediátricos trasplantados de riñón, especialmente si recibían concomitantemente tratamiento con bloqueantes de los canales del calcio (13). El objetivo del presente estudio, ha sido determinar con un ensayo clínico aleatorio a doble ciego el efecto del MNZ sobre el AG incipiente secundario a tratamiento con CsA (Sandimmum ${ }^{\star}$ ) en pacientes adultos trasplantados renales, utilizando como medio de valoración cuantitativa del AG técnicas de análisis de imagen asistidas por ordenador (14).

\section{PACIENTES, MATERIAL Y MÉTODOS}

Se realizó un ensayo clínico aleatorio controlado a doble ciego para determinar el efecto del tratamiento con MNZ sobre el AG incipiente inducido por CsA. Todos los pacientes procedían de nuestro hospital y el estudio recibió la aprobación del Comité Etico de Ensayos Clínicos.
Los criterios de inclusión para este ensayo clínico fueron: a) ser receptor de un trasplante renal; b) ser mayor de 18 años; c) que el tiempo transcurrido desde el trasplante fuera superior a 6 meses e inferior a 2 años; d) Recibir como tratamiento supresor CsA; e) Conservar al menos los 4 incisivos de la arcada dentaria superior e inferior; f) No haber recibido tratamiento periodontal inmediatamente antes o durante el período de estudio; g) haber suministrado a todos los pacientes el consentimiento informado para participar en el ensayo. Fueron criterios de exclusión la sospecha clínica de rechazo del injerto o la ingesta de fármacos con interacción farmacológica con el factor de intervención (MNZ).

De los 34 pacientes que constituían el estudio inicial, ocho pacientes fueron excluidos (2 pacientes tomaban dicumarínicos (Sintron ${ }^{\circledR}$ ) que interaccionan con el MNZ; 2 pacientes presentaban gingivitis aguda; en 3 pacientes faltaban algunos de los incisivos anteriores y l paciente recibió tratamiento odontológico inmediatamente antes de comenzar el estudio. Los 26 pacientes que constituyeron el grupo final, fueron asignados al azar al grupo experimental o al grupo placebo, sacando una bola de una bolsa. El grupo experimental constituido por 13 pacientes (MNZ, $\mathrm{n}=13$ ) recibió $250 \mathrm{mg}$ metronidazol 3 veces/día durante 7 días y el grupo placebo $(n=13)$ recibió una cápsula de placebo 3 veces/días durante 7 días.

La exploración oral de todos los pacientes fue llevada a cabo por dos observadores (FLMA, PJ).

La media de edad de los pacientes fue 42.5 años (rango 24-60). Para cada paciente fueron recogidos los siguientes datos: índices de higiene oral, índice de placa (O'leary) e índice de sangrado (Ainamo y Bay), valoración clínica del AG siguiendo los criterios de Pernu (15), índice morfométrico (IAG) (14), sexo, edad, tiempo transcurrido desde el trasplante, tratamiento con bloqueantes de los canales del calcio, función renal (creatinina sérica y aclaramiento de creatinina) y niveles sanguíneos de CsA.

Al comienzo del estudio, se tomó una fotografía intraoral a cada paciente empleando un abrebocas desechable y una cámara F-3 (Nikon, Tokyo, Japan) con teleobjetivo de $120 \mathrm{~mm}$ y flash anular (Nikon), a una distancia constante de $0.45 \mathrm{~m}$. La película empleada fue en blanco y negro (Ilford, Cheshire, UK) de 100 asa y la positivización se realizó en papel grado 3 politemizado de 13 x 9 (Ilford). Treinta días después, fue repetida la fotografía intraoral en todos los pacientes bajo las mismas condiciones.

Las modificaciones producidas por el AG fueron valoradas por medio de análisis digital de imagen (ADI). Con esta intención, las fotografías en blanco y negro 
fueron digitalizadas usando un escáner Agfa Arcus II con el programa Arcus Bonus (Agfa, Barcelona). La cuantificación fue realizada por medio del programa de análisis de imagen Visilog 4.1 (Noesis CORP., Velizy, France) en entorno Windows 95 (Microsoft Corporation, Redmond, Washington, USA). Las superficies medidas al inicio y final del estudio fueron el área gingival $(\mathrm{AG})$ y área dental $(\mathrm{AD})$. La relación entre ellas fue considerada como el índice de agrandamiento gingival $(\mathrm{IAG}=\mathrm{AG} / \mathrm{AD})$, siguiendo la metodología previamente descrita por nuestro grupo (14). La cuantificación automática de las áreas seleccionadas por ADI nos permitió establecer objetivamente las modificaciones postratamiento.

Los datos obtenidos fueron exportados a la base de datos RSigma Babel (Horus, Harware, Barcelona). Para comprobar si las variables se ajustaban a una distribución normal, se empleo el test de Kolmogorov Smirnov. Una vez comprobado, se aplicaron los tests estadísticos de análisis de la varianza de una vía (Anova) y de comparación de medias para muestras independientes ( $t$ de Student).

\section{RESULTADOS}

La tabla 1 muestra los valores y distribución de algunas de las variables clínicas, morfométricas y analíticas estudiadas. La media de edad y la distribución por sexo fueron similares en los dos grupos (39.1 \pm 11 años el el grupo MNZ frente a $43.3 \pm 10$ en el grupo placebo) (Tabla 1). Respecto a la función renal al comienzo del estudio, los pacientes del grupo MNZ presentaban valores de creatinina sérica significativamente mayores que el grupo placebo $(3.64 \pm 2.63$ frente a $1.60 \pm 0.57$ respectivamente, $\mathrm{p}<0.05 \mathrm{t}$ de Student), los cuales disminuyeron después de 30 días de tratamiento (Tabla l). Los niveles séricos de CsA fueron similares en ambos grupos y en todos los casos se encontraron dentro de rango terapéutico (grupo MNZ $170.5 \pm 27$ frente a grupo placebo $175.8 \pm 29$ ).

El grado de salud oral en los dos grupos fue similar al comienzo del estudio. Los 2 pacientes con gingivitis aguda fueron descartados del estudio para evitar interferencia en los resultados, ambos presentaban enrojecimiento mucoso en sectores gingivales anteriores y ligero aumento del tamaño de las papilas interdentales. El resto de pacientes mostraban una deficiente salud oral con índices intermedios pero con amplio rango (media del índice de placa: $51.76 \%$, rango 10 - 100\%; media del índice de sangrado $54.57 \%$, rango $0-100 \%$ ). El grupo MNZ mostró una ligera disminución de los índices de salud oral después del tratamiento (media del índice de placa: $35.61 \%$, media del índice de sangrado: $42.45 \%$ ), mientras que en el grupo placebo no se observó disminución (media del índice de placa: $53.23 \%$, media del índice de sangrado: $56.35 \%$ ).

La distribución de los pacientes según el AG valorado clínicamente fue el siguiente: El 30\%, no presentaba signos clínicos de AG; el 46.1\%, presentaba AG grado $1 ;$ el $23.9 \%$, AG grado 2 . Ninguno de los pacientes presentaba AG grado 3 (Tabla 1). Al inicio del estudio, las puntuaciones globales del IAG en función del AG clínico presentaron los siguientes valores: encías sin sobrecrecimiento (IAG $0.846 \pm 0.15$ ), AG grado l (IAG $0.944 \pm 0.19$ ), AG grado 2 (IAG $1.238 \pm 0.34$ ).

El IAG obtenido a tiempo cero fue en el grupo MNZ $0.90 \pm 0.16$ y en el grupo placebo $1.11 \pm 0.33$ tras someter a los pacientes a tratamiento y transcurridos

\begin{tabular}{|c|c|c|c|c|c|c|c|}
\hline $\mathbf{P}$ & $\begin{array}{c}\text { MNZ } \\
(n=13)\end{array}$ & $\begin{array}{l}0 \text { DIAS } \\
\text { Placebo } \\
(\mathrm{n}=13)\end{array}$ & $\begin{array}{l}\text { n. total } \\
(\%)\end{array}$ & $\begin{array}{c}\text { MNZ } \\
(\mathrm{n}=13)\end{array}$ & $\begin{array}{c}30 \text { DIAS } \\
\text { Control } \\
(n=13)\end{array}$ & $\begin{array}{c}\text { n. total } \\
(\%)\end{array}$ & \\
\hline $\begin{array}{l}\text { EDAD } \\
\text { NS } \\
\text { Sexo: } \\
\text { Hombres } \\
\text { Mujeres } \\
\text { Acrandamiento Gincival. }\end{array}$ & $\begin{array}{c}39.1 \pm 11 \\
7 \\
6\end{array}$ & $\begin{array}{c}43.3 \pm 10 \\
6 \\
7\end{array}$ & $\begin{array}{l}13(50 \%) \\
13(50 \%)\end{array}$ & $39.1 \pm 11$ & $43.3 \pm 10$ & $\begin{array}{l}13(50 \%) \\
13(50 \%)\end{array}$ & - \\
\hline $\begin{array}{l}\text { Grado } 0 \\
\text { Grado } 1 \\
\text { Grado } 2 \\
\text { Grado } 3 \\
\text { Creatinina sérica (mg/dL) }\end{array}$ & $\begin{array}{c}4(0.843 \pm 0.19) * \\
7(0.932 \pm 0.18) \\
2(0.910 \pm 0.11) \\
0(-) \\
3.64 \pm 2.63\end{array}$ & $\begin{array}{c}4(0.850 \pm 0.14) \\
5(0.962 \pm 0.22) \\
4(1.408 \pm 0.29) \\
0(-) \\
1.60 \pm 0.57\end{array}$ & $\begin{array}{c}8(30 \%) \\
12(46.1 \%) \\
6(23.9 \%) \\
0(0 \%)\end{array}$ & $\begin{array}{c}4(0.896 \pm 0.23) \\
7(0.926 \pm 0.35) \\
2(1.070 \pm 0.15) \\
0(-) \\
2.90 \pm 1.61\end{array}$ & $\begin{array}{c}4(0.958 \pm 0.14) \\
5(1.121 \pm 0.27) \\
4(1.360 \pm 0.34) \\
0(-) \\
1.56 \pm 0.49\end{array}$ & $\begin{array}{c}8(30 \%) \\
12(46.1 \%) \\
6(23.9 \%) \\
0(0 \%)\end{array}$ & $\begin{array}{l}\text { NS } \\
\text { NS } \\
\text { NS } \\
- \\
p<0.05\end{array}$ \\
\hline $\begin{array}{l}(\mathrm{mg} \% / \mathrm{mL} / \mathrm{min}) \\
\text { Niveles CsA sérica }(\mathrm{ng} / \mathrm{mL})\end{array}$ & $\begin{array}{l}40.12 \pm 21.17 \\
170.50 \pm 27.0\end{array}$ & $\begin{array}{l}74.90 \pm 26.23 \\
175.80 \pm 29.0\end{array}$ & & $\begin{array}{l}41.37 \pm 20.39 \\
168.30 \pm 25.0\end{array}$ & $\begin{array}{l}75.04 \pm 25.11 \\
170.61 \pm 27.0\end{array}$ & & $\begin{array}{l}\mathrm{p}<0.01 \\
\text { NS }\end{array}$ \\
\hline
\end{tabular}

Tabla 1: Datos clínicos, morfométricos y analíticos de los 26 pacientes incluidos en el estudio. 
30 días de evolución, el IAG en el grupo MNZ no se modificó $(0.92 \pm 0.28)$ y en los pacientes que tomaron placebo se incrementó ligeramente (1.18 \pm 0.3$)$, aunque sin llegar a ser estadísticamente significativo. Por lơ tanto no existieron diferencias en el IAG para cada grupo entre el principio y el final del estudio. El mayor incremento del IAG en el grupo placebo dió como resultado una diferencia estadísticamente significativa al comparar el grupo tratado con el grupo placebo a los 30 días (grupo MNZ $0.92 \pm 0.28$ frente a grupo placebo $1.18 \pm 0.3, \mathrm{p}<0.05 \mathrm{t}$ de Student), diferencia que se acentuó cuando se compararon sólo los pacientes de ambos grupos con AG clínico grados 1 ó 2 (grupo MNZ $0.96 \pm 0.31$ frente a grupo placebo 1.24 $\pm 0.31, \mathrm{p}<0.05$ t de Student).

La puntuación del IAG fue menor en el $54.4 \%$ de los pacientes del grupo MNZ tras 30 días de seguimiento, mientras que en el grupo placebo sólo fue menor en el $23.1 \%$ de los pacientes y con una diferencia menor entre el valor a tiempo cero y el obtenido transcurridos 30 días. En ninguno de los pacientes incluidos en el ensayo clínico se obtuvo la remisión completa del AG al finalizar el estudio.

\section{DISCUSIÓN}

El AG inducido por CsA aparece en un porcentaje variable (20-70\%) de los pacientes sujetos a esta terapia (1). En el presente estudio, AG leve o moderado ha sido observado en el $70 \%$ de los 26 pacientes portadores de trasplante renal con seguimiento superior a 12 meses, evaluados consecutivamente por especialistas en periodoncia. Este dato revela la necesidad de controlar el estado periodontal de estos pacientes, descrito por Seymour y colaboradores como el único factor de riesgo en el que el periodoncista puede influenciar (16).

La patogenia del AG inducido por CsA no ha sido totalmente establecida, pero se reconoce su carácter multifactorial y donde los fenómenos inflamatorios juegan un importante papel $(17,18)$. Aproximadamente el $40 \%$ del agrandamiento inducido por CsA puede ser considerado de naturaleza inflamatoria (3) y ha sido hipotetizado que el agrandamiento fibrogénico debido al fármaco podría ser magnificado por un componente edematoso lo que produciría un aparente incremento del AG valorado clínicamente (3). En los pacientes trasplantados, la lámina propia gingival invariablemente contiene infiltrado inflamatorio, a pesar del bloqueo de la respuesta inmunológica. La intensidad del infiltrado inflamatorio (CD45-positivo) está relacionada de forma estadísticamente significativa con la severidad del AG (14). El incremento de macrófagos (CD68-positivos) en la lámina propia está también relacionado con la severidad del AG (14). Los macrófagos son los principales productores de TGFBl, La citocina responsable de la respuesta hiperplásica fibrosa. Además, la CsA induce alteración en el perfil de secreción de citocinas por el tejido gingival y del infiltrado inflamatorio (incremento de interleucinas IL-1ß, IL-6), que causan disregulación de la remodelación del tejido conectivo y acumulación de los componentes de la matriz extracelular (19).

La mayoría de los estudios han mostrado asociación entre el estatus de higiene oral y la prevalencia y severidad del agrandamiento gingival inducido por fármacos (1). Esto sugiere que la inflamación gingival inducida por la placa bacteriana puede tener un importante papel en el desarrollo y la expresión de los cambios gingivales. Los cambios observados en el contorno del AG inducido por fármacos pueden estar en parte exacerbados por la inflamación ocasionada por la placa bacteriana (17). Por todo lo expuesto, podrían explicarse los mejores resultados obtenidos cuando se han aplicado tratamientos para mejorar la higiene oral junto a terapia antibiótica. En nuestro estudio, seguramente si hubiéramos eliminado los irritantes locales y la inflamación con raspado e instrucciones sobre higiene oral hubiéramos conseguido mayores reducciones del AG clínico, pero el propósito del estudio era evaluar solamente el efecto del fármaco y por ello no se interfirió en las condiciones periodontales de los pacientes.

El tratamiento antimicrobiano con macrólidos desempeña un prominente papel en la terapia de las infecciones odontogénicas agudas y como tratamiento adjuvante o profiláctico en la cirugía de la infección periodontal asociada a procesos inflamatorios crónicos (20). EL MNZ ha mostrado poseer un importante efecto sobre la flora microbiana subgingival de las lesiones periodontales y después de múltiples dosis, se obtiene una alta concentración en el fluido crevicular, superior a la concentración inhibitoria mínima para la mayoría de los patógenos periodontales (21). Los resultados de nuestro estudio en pacientes adultos trasplantados con AG incipientes indican que el tratamiento con MNZ no induce su remisión completa. Estos hallazgos contradicen los resultados de Wong (4), que informó sobre la remisión completa en cuatro pacientes con AG, en algún caso severo, y el control de la recurrencia del AG, empleando un protocolo de tratamiento similar al nuestro. Consideramos, que estos pacientes pudieron presentar un AG con un componente inflamatorio insospechadamente intenso o simplemente algún tipo de inflamación o gingivitis en relación con su estatus de inmunosupresión y no verdaderos AG. En concordancia con nuestros resultados, Aufircht y colaboradores (13), han publicado la ausencia de mejoría del AG inducido por CsA entre pacientes trasplantados pediátricos tratados con 
MNZ, especialmente entre los que recibían además tratamiento con bloqueantes de los canales del calcio. Nuestro estudio a doble ciego muestra que el tratamiento con MNZ en un corto período no induce la remisión completa del AG, como queda demostrado por las pequeñas diferencias en el área gingival, comparando la segunda imagen intraoral del grupo tratado con la primera de cada paciente. No obstante, se obtuvo reducción en el IAG en el $54 \%$ de los pacientes tratados con MNZ y mayor progresión del AG en el grupo placebo frente al grupo MNZ de manera estadísticamente significativa. Estos hallazgos sugieren que el efecto antibacteriano del MNZ frena parcialmente el progresivo curso clínico del AG.

El MNZ es metabolizado por el citocromo P450 y es eliminado por vía renal sin interferir con los niveles séricos de CsA (4). En nuestros pacientes, los niveles de creatinina iniciales fueron más elevados que en los sujetos normales y a pesar de que el grupo MNZ fue el que presentó peor función renal, los valores de creatinina y aclaramiento de creatinina no sufrieron cambios significativos al final del estudio, indicando con ello que el MNZ no es nefrotóxico y no interfiere con la función renal a las concentraciones empleadas.

Recientemente, han sido publicados estudios usando azitromicina que obtienen remisiones del AG parciales y en ocasiones completas (6-9). La azitromicina pertenece a una nueva clase de macrólidos semisintéticos denominados azalidas derivados de la eritromicina, que no afectan al citocromo P450 hepático y no modifica la farmacocinética de otros fármacos, especialmente de la CsA (22). Tras la ingestión oral, la azitromicina puede ser detectada en tejido gingival durante 7 a 10 días (20) y la concentración en saliva es 10 a 100 veces mayor que en el plasma (23). Además, la azitromicina rápidamente penetra en fibroblastos y macrófagos, donde la concentración es 100 a 200 veces mayor que la encontrada en la matriz extracelular (24).

Palomar y colaboradores (7) en 10 pacientes trasplantados renales con AG tratados con azitromicina, obtuvieron cinco casos de remisión completa del agrandamiento y reducción de más del $50 \%$ en los otros cinco pacientes. Nowicki publicó la regresión parcial de AG en un caso severo (grado 3) en un paciente trasplantado renal inmunosuprimido con CsA tras tres días de tratamiento con azitromicina, aunque la repetición del tratamiento después de tres meses no produjo nueva reducción del agrandamiento (9). Ninguno de estos estudios han empleado grupo control para establecer el verdadero efecto sobre el AG de la azitromicina y son descripciones clínicas sin una exhaustiva valoración de la cavidad oral y sin estudio biópsico para verificar las modificaciones de las lesiones histopatológicas. En cualquier caso, nosotros creemos que en todos los casos descritos anteriormente tratados con azitromicina, la reducción del AG está relacionado con la disminución de la intensidad del proceso inflamatorio acompañante, más que con la reabsorción o remodelación de la fibrosis que tiene lugar en la lámina propia gingival, que en ningún caso ha sido testificada.

En conclusión, el tratamiento durante siete días con MNZ no produce la regresión completa del AG inducido por CsA, pero puede detener su progresión clínica, probablemente a través de la reducción de la sobreinfección bacteriana e inflamación gingival.

\section{AGRADECIMIENTOS}

A la Sra. Inmaculada Morillas por la obtención de las fotografías intraorales.

\section{ABSTRACT}

Background: Drug-induced gingival overgrowth (GO) remains a significant problem for the periodontologist. Partial and total regressions of this GO have been reported after a short course of antibiotics.

Methods: We conducted a double-blind controlled randomized study in order to determine the effect of 7 days of metronidazole treatment $(250 \mathrm{mg} / 3$ times day) on the regression of incipient cyclosporin $A$ induced GO in adult renal transplanted patients. The quantitation of the GO was performed with Image Digital Analysis.

Results: At the end of the study, none of the 26 patients in the trial showed complete remission of the GO. Before treatment, the GO index was $0.90 \pm 0.16$ in the patients treated with metronidazole (MNZ group $=13$ ) and $1.11 \pm 0.33$ in the controls (placebo group =13). At the end of the study (30 days) the GO index score was lower in $54.4 \%$ of the MNZ and the difference between groups was statistically significant (MNZ $0.92 \pm 0.28$ vs. placebo $1.18 \pm 0.3, \mathrm{p}<0.05$ Student's t test.

Conclusions: The results of our present study indicate that a 7-day course of metronidazole does not induce the complete remission of $\mathrm{CsA}$-induced gingival overgrowth but can partially abate the progression of the GO, probably through a decrease in the bacterial overinfection and gingival inflammation.

\section{KEY WORDS}

Metronidazole, cyclosporin A, gingival overgrowth. 


\section{CORESPONDENCIA}

Francisco Luis Mesa Aguado

Avda. Medina Olmos n $22,1^{\circ} \mathrm{B}$

18500 Guadix-Granada. España

Telf.: 958663132

FAX: 958663132

e-mail: fmesa@ugr.es

\section{BIBLIOGRAFÍA}

1. Daley TD, Wysocki GP: Clinical and pharmacologic correlations in cyclosporine-induced gingival hyperplasia. Oral Surg Oral Med Pathol 1986;62:417-421.

2. Hassell TM, Hefti AF. Drug-induced gingival overgrowth: Old problem. Crit Rev Oral Biol Med 1991;2:103-107.

3. Kantarci A, Cebeci I, Tuncer Ö, Çarin M, Firatli E. Clinical effects of periodontal therapy on the severity of cyclosporin A-induced gingival Hyperplasia. J Periodontol 1999;70:587-593.

4. Wong W, Hodge MG, Lewis A, Sharpstone P, Kingswood JC. Resolution of cyclosporin-Induced gingival hypertrophy with metronidazole. The Lancet 1994;343:989.

5.Wahlstrom E, Zamora JU, Teichman S. Improvement in cyclosporin-associated gingival hyperplasia with azithromycin therapy. N Engl J Med 1995;332:153-754.

6. Gomez E, Sanchez-Nuñez M, Sanchez JE, et al. Treatment of cyclosporin-induced gingival hyperplasia with azithromycin. Nephrol Dial Transplant 1997;12:2694-2697.

7. Palomar R, Belart M, Soy D, Oppenheimer F, Campistol JM. Effectiveness and safety of azithromycin in the treatment of cyclosporine-induced gingival overgrowth. Nephron 1998;79(1):101-102.

8. Jucgla A, Moreso F, Sais G, Gil-Vernet S, Graells J, Grinyo JM, Peyri J. The use of azithromycin for cyclosporin-induced gingival overgrowth Br J Dermatol 1998;138:188-189.

9. Nowicki M, Kokot F, Wiecek A. Partial regression of advanced cyclosporin-induced gingival hyperplasia after treatment with azithromycin. A case report. Ann Transplant 1998;3:25-27.

10. Lesche WJ, Grossman N, Giordano J. Metronidazole in periodontitis. J Clin Periodontol 1993;20:96-104.

11. Pavicic MJAMP, van Winkelhoff AJ, Douqué NH, Steures RWR, de Graaff J. Microbiological and cliical effects of metronidazole and amoxicillin in Actinobacillus actinomicetemcomitans-associated periodontitis. J Clin Periodontol 1994;21:107-112.
12. Rotzetter PA, Le Liboux A, Pichard E, Cimasoni G. Kinetics of spiramycin/metronidazole (Rodogyl) in human gingival crevicular fluid, saliva and blood. J Clin Periodontol 1994;21:595-600.

13. Aufricht C, Hogan EL, Ettenger RB. Oral metronidazole does not improve cyclosporine $\mathrm{A}$-induced gingival hyperplasia. Pediatr Nephrol 1997;11:552-555.

14. O'Valle F, Mesa F, Aneiros J, et al. Gingival overgrowth induced by nifedipine and cyclosporin a. Clinical and morphometric study image analysis. J Clin Periodontol 1995;22:591-597.

15. Pernu HE, Pernu LMH, Knuuttila MLE, Huttunen KRH. Gingival overgrowth among renal transplant recipients and uraemic patients. Nephrol Dial Transplant 1993;8:1254-1258.

16. Seymour RA, Ellis JS, Thomason JM. Risk factors for druginduced gingival overgrowth. J Clin Periodontol 2000;27:217-223.

17. Seymour RA, Thomason JM, Ellis JS. The pathogenesis of drug-induced gingival overgrowth. J Clin Periodontol 1996;23:165-175.

18. Mesa FL, López C, Moreno E, O'Valle F. Crecimientos gingivales inducidos por medicamentos. Periodoncia 1994;4:177-186.

19. Myrillas TT, Linden GJ, Marley JJ, Irwin CR. Cyclosporin A regulates interleukin- $1 B$ and interleukin- 6 expression in gingiva: implications for gingival overgrowth. J Periodontol 1999;70:294-300.

20. Blandizzi C, Malizia T, Lupetti $\AA$, et al. Periodontal tissue disposition of azithromycin in patients affected by chronic inflammatory periodontal diseases. J Periodontol 1999;70:960-966.

21. Van Oosten MAC, Notten FJW, Mikx FHM. Metronidazole concentrations in human plasma, saliva, and gingival crevice fluid after a single dose. J Dent Res 1986;65:1420-1423.

22. Amacher DE, Schomacker SJ, Retsema JA. Comparison of the effects of the new azalide antibiotic, azithromycin estolate on rat liver cytochrome P-450. Antimicrob Agents Chemother 1991;35:1186-1190.

23. Foulds G, Johnson RB. Selection of dose regimens of azithromycin. J Antimicrob Chemother 1993;31(suppl. E):39-50.

24. Gladue RP, Snyder ME. Intracellular accumulation of azithromycin by cultured human fibroblasts. Antimicrob Agents Chemother 1990;34:1056-1060. 